\title{
STRIPPING FOILS FOR MULTITURN CHARGE EXCHANGE INJECTION INTO THE FERMILAB BOOSTER
}

C. Hojvat, M. Joy, R. C. Webberk

\section{SUMMARY}

Carbon foils with surface densities of $200 \mu \mathrm{gcm}^{-2}$ were selected as the stripping medium for the charge exchange injection system into the Fermilab Booster accelerator. These foils have extremely long lifetimes. The criteria leading to their selection and the preparation method are discussed.

\section{INTRODUCTION}

Multiturn Charge Exchange Injection (CEI) recently became operational in the rapid cycling Fermi lab Booster accelerator. 1 The CEI method is based on the capture of protons by stripping electrons from $\mathrm{H}^{\circ}$ atoms or $\mathrm{H}^{-}$ions in the closed orbit of a cyclic accelerator.

For operation with CEI at Fermilab, $\mathrm{H}^{-}$ions are accelerated in the Linac to the Booster injection energy of $200 \mathrm{MeV}$ with currents of up to $43 \mathrm{~mA}$. Injection into the Booster can take place for up to $140 \mu \mathrm{s}$, the equivalent of 50 turns. Emittances of the $H^{-}$beam are of the order of $8 \pi \times 10^{-6} \mathrm{~m}$ for both planes.

Photodetachment, dissociation in magnetic fields and charge exchange in gases, organic and inorganic foils were studied as possible stripping methods. ${ }^{2}$ CEI at the Argonne ZGS utilizes organic films for charge exchange. ${ }^{3}$ The films have an unpredictable lifetime in the beam and require frequent replacement. We have finally considered only carbon foils and metallic films as potential candidates.

\section{CHOICE OF MATERIAL}

Charge exchange phenomena give rise to capture or loss of electrons by the $\mathrm{H}^{-}$ions traversing the foil. At our energy electron capture can be neglected. Electron loss cross sections increase with atomic number and stripping efficiency increases with foil thickness.

For multiturn CEI, the circulating bean must traverse the foil for at least as many times as the number of turns injected. Multiple scattering in the foil results in emittance blow-up of the circulating beam. The mean squared scattering angle increases with the atomic number of the scatterer and the thickness of material.

The expression for the stripping efficiency as a function of the electron loss cross sections $\sigma_{-10}$ and $\sigma_{01}$ can be found elsewhere. ${ }^{2}$ Both cross sections have been measured up to $15 \mathrm{MeV}^{4}$ and $\sigma_{01}$ up to $45 \mathrm{MeV}$. $^{5}$. The values for $\sigma_{-1} 10$ were extrapolated to $200 \mathrm{MeV}$ assuming the theoretical dependence on the velocity. ${ }^{6}$ Extrapblation of $\sigma_{01}$ assumed an $E^{-k}$ dependence, $E$ being the kinetic energy of the protons, with $k=0.93$ for helium, $b .84$ for nitrogen and 0.82 for argon. For elements with atomic number $Z \leq 18$ the values at $200 \mathrm{MeV}$ were obtained by interpolation with the fits:

$$
\begin{aligned}
& b_{-10}=(-0.174+0.283 \mathrm{z}) 10^{-18} \mathrm{~cm}^{2} / \text { atom } \\
& b_{01}=\left(-0.120+0.101 z+4.32 \times 10^{-3} \mathrm{z}^{2}\right) 10^{-18} \mathrm{~cm}^{2} / \text { atom }
\end{aligned}
$$

\footnotetext{
Fermi National Accelerator Laboratory, P. 0. Box 500, Batavia, Illinois 60510 .

ropter aceu by Uilversicles nesear cll Assulaliull, Iric., under contract with the U. S. Department of Energy.
}

Figure 1 shows the calculated stripping efficiency as a function of the foil thickness for elements lighter than argon which can be formed into thin foils.

The mean squared scattering angle can be expressed in terms of the radiation length of the foil material and its thickness. 7 Figure 2 shows the correlation of the calculated stripping efficiencies as a function of the mean squared scattering angle. The lower, the atomic number, the lower the multiple scattering for a given stripping efficiency.

Commercial suppliers of thin foils were contacted for availability and cost of $3.8 \mathrm{~cm} \times 7.6 \mathrm{~cm}$ foils of sufficient thickness to obtain stripping efficiencies in excess of $70 \%$, with the following response:

$\begin{array}{llr}\text { Material } & \text { Thickness }(\mu \mathrm{m}) & \text { Cost/Foil } \\ \text { Lithium } & 2.7 \text { to } 10 & 250.00 \\ \text { Beryllium } & 0.6 \text { to } 2.4 & 400.00 \\ \text { Carbon } & 0.5 \text { to } 2.0 & 23.00 \\ \text { Aluminum } & 0.4 \text { to } 1.5 & .75 .00\end{array}$

Both lithium and beryllium foils are expensive anc present handiing problems. The choice is left between carbon and aluminum. Carbon not only gives rise to lower multiple scattering but is also cheaper than aluminum.

Systematic studies of thin carbon foils lifetimes are available in the literature. ${ }^{8}$ The measured lifetimes are consistent with depending on the energy deposited per unit volume by the passing ion and independent of foil thickness. We then assume that data for heavy ions, thinner foils and lower currents can be extrapolated to our application. For bombardment with $200 \mathrm{MeV}$ ions we estimate a lifetime of $8.9 \times 10^{3} \mu \mathrm{A}$ min $\mathrm{mm}^{-2}$. This corresponds to $1.27 \times 10^{21}$ protons over an area of $380 \mathrm{~mm}^{2}$ or two years of operation with 5 turn injection at present intensities.

Carbon foils $200 \mu \mathrm{gcm}^{-2}$ thick were chosen as the stripping medium for CEI into the Fermilab Booster, with an expected stripping efficiency of $98 \%$.

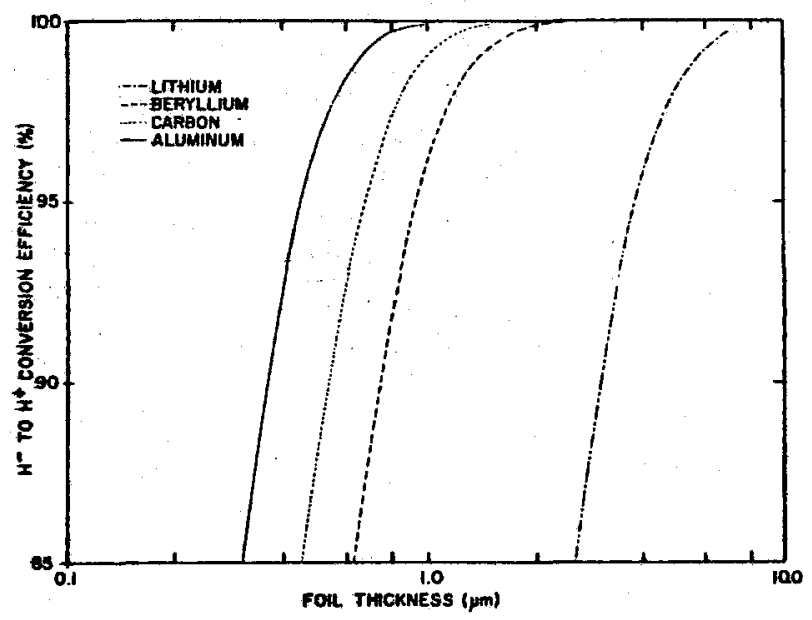

riy. 1. 3hrippling erriliemy vs. rull LILKness for low 2 elements. 


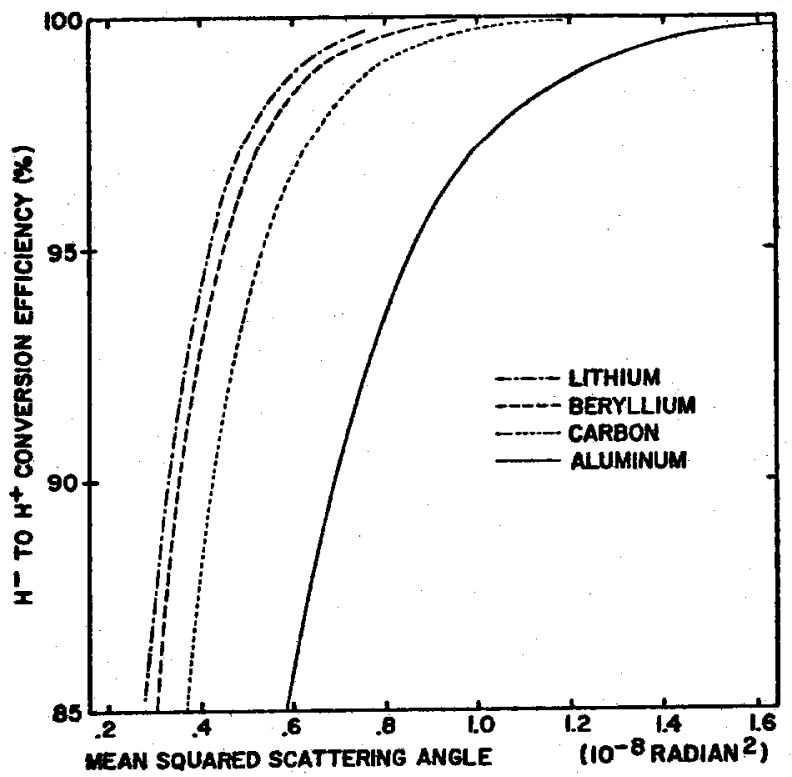

Fig. 2. Stripping efficiency vs. mean squared scattering angle for low $Z$ elements.

\section{EMITTANCE GROWTH}

The emittance growth of a beam due to scattering in a stripping foil during multiturn CEI has been discussed theoretically by Cooper and Lawrence.9. For a normally distributed beam in transverse phase space, the change in the emittance $\Delta E$ of the circulating beam after $\mathrm{N}$ injected turns is given by,

$$
\Delta E=\frac{1}{2} B_{0} N \times\left[\sigma_{c_{\cdot}}\left(\overline{\delta y^{\prime}}\right)^{2}\right] \pi
$$

$B$ is the value of the $\beta$ function of the machine at the injection point, $X$ is the foil thickness, and the quantity in brackets is the product of the effective coulomb cross section for a collision and the mean square of a component of the angle caused by such a collision. The emittance growth is proportional to the number of foil traverses, and not to the square root as one would expect from the usual multiple scattering formula."

To obtain the numerical value of $\left[\sigma_{c} \cdot \overline{\left(\delta y^{\prime}\right)^{2}}\right]$ we fol $w$ the treatment by Courant. ${ }^{10}$ The maximum angle for integrating the coulomb cross section is taken as the one given by the machine acceptance. For larger angles, particles will be lost and do not remain in the machine to contribute to the emittance growth. In the Fermilab Booster the maximum angles are $9.0 \times 10^{-4} \mathrm{rad}$ vertically and $2.0 \times 10^{-3}$ rad horizontally. With these angles we obtain:

$$
\begin{aligned}
& {\left[\sigma_{c \cdot}\left(\overline{\left.\delta y^{\prime}\right)^{2}}\right]_{H}=2.68 \times 10^{-28} \mathrm{rad}^{2} \mathrm{~cm}^{2} \text { atom }^{-1}\right.} \\
& {\left[\sigma_{c \cdot}\left(\overline{\left.\delta y^{\prime}\right)^{2}}\right]_{V}=2.21 \times 10^{-28} \mathrm{rad}^{2} \mathrm{~cm}^{2}\right. \text { atom }}
\end{aligned}
$$

For a $200 \mu \mathrm{gcm}^{-2}$ foil the relative growths per injected turn given by (1) are, $0.3 \%$ vertically and $0.1 \%$ horizontally, for initial beam emittances of $8 \pi x$ $10^{-6} \mathrm{~m}$. The expected emittance growth is negligible provided that the circulating beam does not remain on the foil for many turns after injection is completed.

\section{CARBON FOILS}

The dimensions of the free area of the stripping foil must be large enough so that no circulating beam can strike the foil holder. The " $C$ " shaped frame witl the dimensions indicated in Figure 3 is utilized for mounting the foils. The unsupported area of the mount ed foils is about $16 \mathrm{~cm}^{2}$. To reduce emittance growth due to multiple scattering the circulating beam is moved off the foil after injection is completed. The free edge of the foil provides an obstruction-free path for the beam to move across.

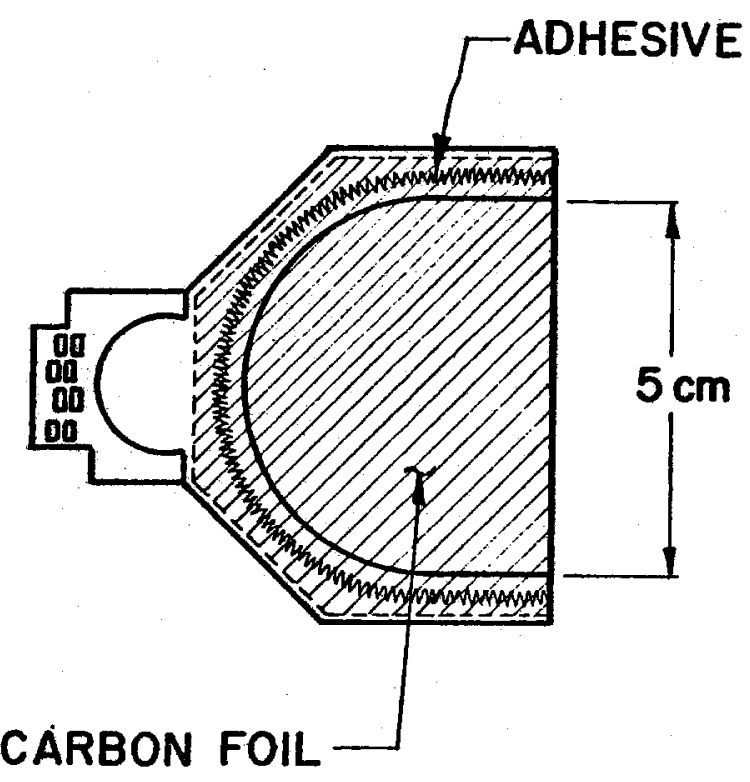

Fig. 3. Frame for Carbon stripping foils.

Carbon foils are purchased commercially as a $50 \mathrm{~mm} \times 70 \mathrm{~mm}$ film evaporated onto a glass slide. 11 The outer contour of the frame is cut on the film with a razor blade and a template. One of the original edges of the film becomes the free edge of the foil. Separation of the film from the glass is accomplished by surface tension in a room temperature bath of distilled water. The foil floats on the water surface and is lifted on a frame which has been coated with an adhesive. The final separation of the foil from the water is performed with the frame in the vertical posi tion, breaking the surface tension by first lifting on side of the frame from the water. After mounting, the foils are left to dry and then stored under vacuum.

While thin foils adhere to clean metal surfaces, our experience with thicknesses greater than $100 \mu \mathrm{gcm}^{-}$ indicates that an adhesive is required. Both sodium silicate, "water glass", and Eastman $910^{12}$ were tried. The former shrinks when drying, having a tendency to break the edge of the foils, whereas Eastman 910 has proven satisfactory.

Initially foils are very flexible and fragile. As they are exposed to beam they become more rigid and much less fragile, with a tendency to grow on the transverse dimensions, bulging out of the plane of the supporting frame.

\section{RESULTS}

During the docion ctane of the multiturn CFT custem, carbon foils were exposed to the circulating beam 
in the Booster. No tested foil suffered beam induced damage and an expected life time of 120 hours minimum was projected.

With the CEI system now operational since March 10, 1978, long term foil exposures have been achieved. Since then, 3 foils have been utilized during operation:

\begin{tabular}{|c|c|c|c|c|}
\hline Foil \# & $\begin{array}{l}\mathrm{H}^{-} \text {Injegcted } \\
\times 1018\end{array}$ & $\begin{array}{l}\text { Protons on } \\
\text { Foil } \times 10^{19}\end{array}$ & $\begin{array}{l}\text { Date } \\
\text { from }\end{array}$ & to \\
\hline 1 & 6.0 & 7.8 & $3 / 21 / 78$ & $7 / 10 / 78$ \\
\hline 2 & 6.4 & 8.3 & $7 / 10 / 78$ & $10 / 23 / 78$ \\
\hline 3 & 17.0 & 22.1 & $12 / 11 / 78$ & present \\
\hline
\end{tabular}

None of these foils suffered beam induced damage or breakage. Foil \#1, shown in Figure 4, was saved for display at. Fermilab. Foil \#2 had an unfortunate encounter with a vacuum cleaner. Foil \#3 has been in operation since December 11, 1978.

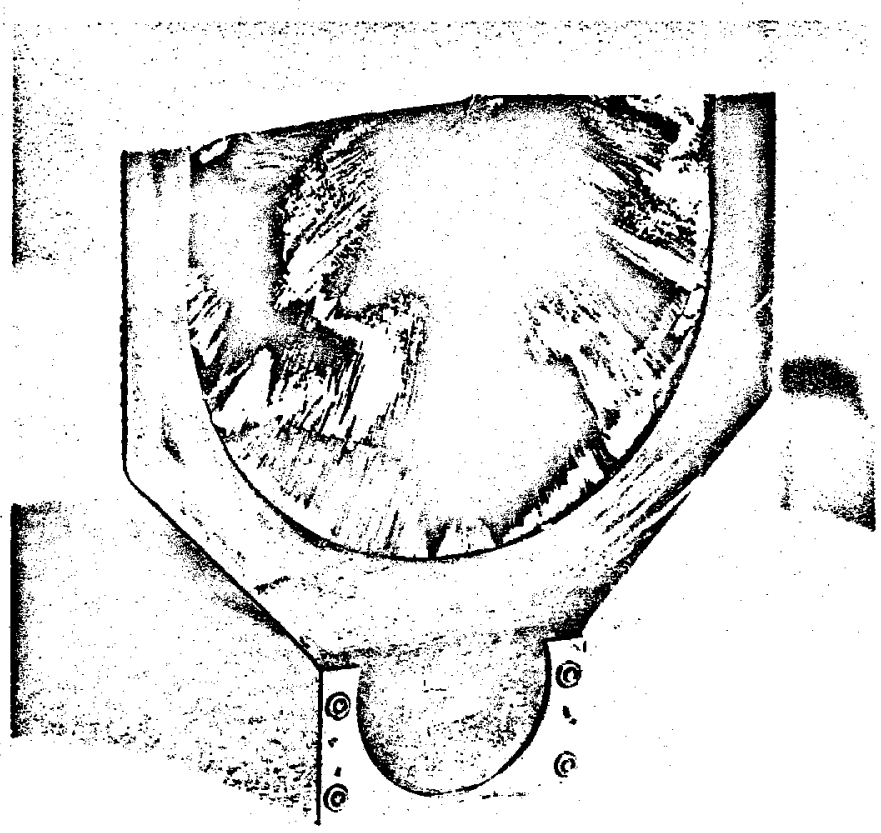

Fig. 4. Photograph of foil \#1 after $6.0 \times 10^{18}$ injected $\mathrm{H}^{-}$ions.

In conclusion, an efficient, reliable and troublefree solution to stripping was found for multiturn charge exchange injection into the Fermilab Booster accelerator.
The authors would like to acknowledge the support from their colleagues in the Booster group, especiall] that of $R$. P. Johnson during the initial phase of this work, D. Cosgrove for engineering support and

T. Schmitz for design work.

\section{REFERENCES}

1. C. Hojvat et al., "The Multiturn Charge Exchange Injection System for the Fermilab Booster Accelerator" this conference.

2. M. Joy, Fermilab TM-699, 1976; C. Hojvat, Internal Memorandum.

3. C. W. Potts, IEEE, NS-24, p. 1385, 1977.

4. H. Tawara and A. Russek, Review of Modern Physics, 45, p. 178, 1973.

5. E. Acerbi et a]., Lettere al Nuovo Cimento, 10, p. $598,1974$.

6. G. H. Gillespie, Physical Review, 16, p. 943, 1977.

7. Particle Data Group, Physics Letters, 75B, No. 1, 1978.

8. A. E. Livingston et al., Nuclear Instruments and Methods, 148, p. 125, 1978.

9. R. K. Cooper and G. P. Lawrence, IEEE, NS-22, p. $1916,1975$.

10. E. D. Courant, Review of Scientific Instruments, 24, p. 836, 1953.

11. Arizona Carbon Foil Co., Arizona.

12. Eastman Chemical Products, Inc., Tennessee. 